\title{
Emerging roles of exosomes in neuron-glia communication
}

\section{Carsten Frühbeis, Dominik Fröhlich and Eva-Maria Krämer-Albers*}

Department of Molecular Cell Biology, Johannes Gutenberg University Mainz, Mainz, Germany

\section{Edited by:}

Claudia Verderio, CNR Institute of Neuroscience, Italy

\section{Reviewed by:}

Felipe A. Court, Pontificia Universidad Catolica de Chile, Chile

Rémy Sadoul, Institut National de la

Santé et de la Recherche Médicale,

France

\section{${ }^{*}$ Correspondence:}

Eva-Maria Krämer-Albers, Department

of Molecular Cell Biology, Johannes

Gutenberg University Mainz,

Bentzelweg 3, 55128 Mainz,

Germany.

e-mail:emkraemer@uni-mainz.de
Brain function depends on coordinated interactions between neurons and glial cells. Recent evidence indicates that these cells release endosome-derived microvesicles termed exosomes, which are 50-100 nm in size and carry specific protein and RNA cargo. Exosomes can interact with neighboring cells raising the concept that exosomes may mediate signaling between brain cells and facilitate the delivery of bioactive molecules. Oligodendrocytes myelinate axons and furthermore maintain axonal integrity by an yet uncharacterized pathway of trophic support. Here, we highlight the role of exosomes in nervous system cell communication with particular focus on exosomes released by oligodendrocytes and their potential implications in axon-glia interaction and myelin disease, such as multiple sclerosis. These secreted vesicles may contribute to eliminate overproduced myelin membrane or to transfer antigens facilitating immune surveillance of the brain. Furthermore, there is emerging evidence that exosomes participate in axon-glia communication.

Keywords: microvesicles, exosomes, neuron-glia communication, oligodendrocytes, axon-glia interaction, myelin disease

\section{INTRODUCTION}

Glial cells actively participate in brain development and function necessitating communication between neurons and glia (Allen and Barres, 2009). Their functions range from metabolic support to myelination, immune defense, and engagement in synapse formation and plasticity. Oligodendrocytes ensheath axons with an insulating myelin sheath facilitating electric impulse propagation. Myelination requires intense communication between oligodendrocytes and neurons, which is also essential for the maintenance of axonal integrity over the lifetime (Nave, 2010a).

Recent reports describe the horizontal transfer of biomolecules by secreted extracellular vesicles, which is increasingly becoming established as a general mode of intercellular communication (Simons and Raposo, 2009; Camussi et al., 2010). Neurons and the major types of glia release vesicles, raising the possibility that communication mediated via extracellular vesicles is a common mechanism in the CNS. Small vesicles, here referred to as microvesicles, shed directly from the plasma membrane or originate from the endosomal system (Lakkaraju and RodriguezBoulan, 2008; Cocucci et al., 2009). A mixed population of such vesicles has been detected in body fluids including cerebrospinal fluid (Vella et al., 2008). Exosomes are released by fusion of multivesicular bodies (MVBs) with the plasma membrane and secretion of the intraluminal vesicles (ILVs) into the extracellular space. They are $50-100 \mathrm{~nm}$ in diameter and carry specific protein and RNA cargo. Exosomal membranes are enriched in cholesterol and sphingomyelin. Proteins relating to their biogenesis (Alix and Tsg101), distinct cytosolic proteins such as heatshock proteins, and certain membrane proteins (tetraspanins, integrins) are sorted into exosomes whereas others are excluded (for review, see Thery et al., 2009; Thery, 2011, and references therein).
Cells utilize exosomes to dispose of unwanted proteins or to exchange signals with neighboring cells. As an example, erythrocytes remove the transferrin receptor via exosomes during maturation, instead of eliminating it via internal degradation. Proteins implicated in cell interaction are strikingly abundant in exosomes, which thus suggests a role in cell-cell communication. In the immune system, antigen presenting cells (APCs) release exosomes containing MHC and costimulatory molecules to modulate T-cell activation (Thery et al., 2009). Furthermore, microvesicles (including exosomes) transport miRNAs and mRNAs from cell to cell. Translation of microvesicle-derived mRNAs is initiated and new proteins are synthesized. In turn, transferred miRNAs inhibit expression of resident proteins. Thus, shuttled RNAs can alter the proteome of recipient cells (Valadi et al., 2007; Skog et al., 2008; Pegtel et al., 2010; Zhang et al., 2010).

This review describes the characteristics and functions of microvesicles secreted by neurons and glia (collectively referred to as neural cells), with particular focus on exosomes released by oligodendrocytes. We propose a role of oligodendroglial exosomes in axon-glia interaction and hypothesize that they shuttle functional molecules to neurons, thus influencing neuronal properties.

\section{CLASSIFICATION AND GENERAL COMPONENTS OF EXOSOMES}

Exosomes and other microvesicles can be isolated from culture supernatants or body fluids by differential centrifugation and filtration. With current technology, the isolation of extracellular vesicles directly from tissues is impossible because membrane debris and internal vesicles contaminate the preparation. Furthermore, discrimination between different types of microvesicles is difficult. To distinguish exosomes from other secreted vesicles, 
their characteristic size (diameter below $100 \mathrm{~nm}$ ), protein composition, density, and endosomal origin are commonly used criteria. Exosomes are derived from MVBs and correspond to the ILVs, which bud from the limiting membrane into the lumen of late endosomes. ILV-budding involves the action of the ESCRT (endosomal sorting complex required for transport) machinery, though its exact role in exosome biogenesis is not clear and appears celltype dependent (Simons and Raposo, 2009; Bobrie et al., 2011). Anyhow, ESCRT and associated proteins such as Tsg101 and Alix are integrated in exosomes and serve as markers of their identity. In addition, ESCRT independent mechanisms of ILV formation have been described involving sorting of exosome cargo to endosomal domains and ceramide-mediated budding from the limiting membrane (Trajkovic et al., 2008; Buschow et al., 2009). Possibly, distinct endosomal sorting mechanisms lead to the generation of subpopulations of exosomes. MVB trafficking and fusion is regulated by Rab-family GTPases. In oligodendroglial cells, Rab35 regulates docking of MVBs to the plasma membrane (Hsu et al., 2010). Moreover, Rab27a and Rab27b are involved in the docking step in Hela cells (Ostrowski et al., 2010).

Proteomic and microarray analysis on a range of exosome preparations yielded a reproducible compendium of exosomeassociated proteins and RNAs, summarized in the database Exocarta (Mathivanan et al., 2012). Exosomes contain a distinct set of proteins conserved across different cell types and species. Typical proteins are cytoskeletal proteins such as tubulin and actin, heat-shock proteins (Hsp70, Hsp90), metabolic enzymes of the glucose metabolism, Flotillin-1, signal transduction proteins (kinases, heterotrimeric G proteins), MHC molecules, clathrin, proteins involved in transport and fusion (annexins, Rab proteins), and translation elongation factors. Strikingly abundant in exosomes are proteins of the tetraspanin family, e.g., CD9, CD63, CD81, and CD82 (van Niel et al., 2006; Simpson et al., 2008; Thery et al., 2009). Additionally, exosomes contain celltype specific components reflecting the host cell identity and presumably also hinting at the biological function of the released exosomes.

\section{CHARACTERISTICS OF CNS EXOSOMES}

In the CNS, neurons, microglia, astrocytes, and oligodendrocytes have been reported to secrete microvesicles into the extracellular environment. In response to glutamatergic synaptic activity, cultured cortical and hippocampal neurons release microvesicles with the characteristics of exosomes. Neuronal exosomes carry the cell adhesion molecule L1, the GPI-anchored prion protein, as well as the GluR2/3 subunits of the AMPA receptor (Faure et al., 2006; Lachenal et al., 2011). The Parkinson disease related protein $\alpha$-synuclein is secreted from a neuroblastoma cell line by exosomes and can influence the viability of neighboring cells (Emmanouilidou et al., 2010).

Oligodendrocytes release exosomes that include the lipids galactocerebroside, sulfatide, and cholesterol (Krämer-Albers et al., 2007), which are also prominent in oligodendroglial lipid rafts and represent characteristic myelin lipids (Krämer et al., 1997). The proteomic profile of oligodendroglial exosomes mirrors the exosome-pattern of marker proteins (Alix, Tsg101, Flotillin-1), ubiquitous tetraspanins (CD81, CD63), and chaperones (Figure 1). They are furthermore characterized by the presence of unique myelin proteins, such as PLP, CNP, MAG, and MOG. Remarkably, oligodendroglial exosomes carry a range of enzymes such as the NAD-dependent deacetylase sirtuin-2, oxidative stress alleviating peroxiredoxins and
A

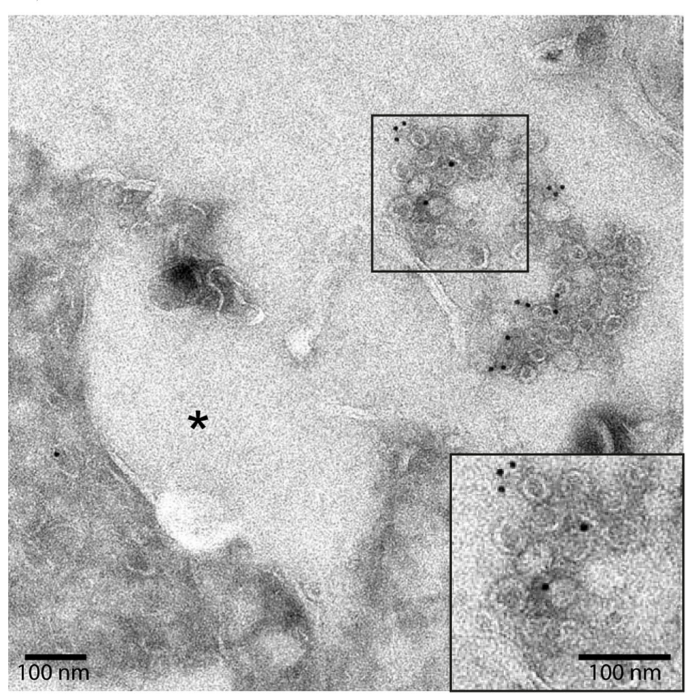

B

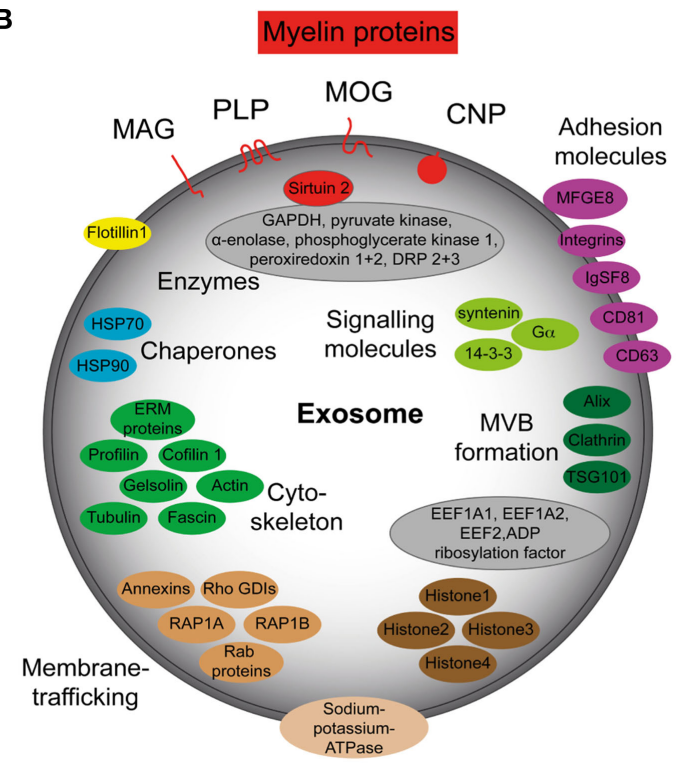

putative MVB fusion profile that resulted in exosome release. Scale bar, $100 \mathrm{~nm}$. (B) Protein composition of a typical oligodendroglial exosome. The illustration is based on proteomic analyses of exosome preparations derived from oligodendroglial cells (Krämer-Albers et al., 2007).
FIGURE 1 | Characteristics of oligodendroglial exosomes. (A) Electron micrograph of exosomes in the extracellular space released by primary oligodendrocytes. Immuno-gold labeling was performed with antibodies recognizing myelin-associated glycoprotein (MAG). Asterisk indicates the 
dihydropyrimidinase-related proteins, and glycolytic enzymes (GAPDH, pyruvate kinase, $\alpha$-enolase).

Microglia are the resident macrophages of the CNS. In pathological situations, they become activated and execute immune functions such as antigen presentation (Kettenmann et al., 2011). Microglia secrete exosomes containing the expected exosomal proteins as well as a set of proteins previously reported for B cell- and dendritic cell-derived exosomes. In addition, they carry the surface-bound aminopeptidase N (CD13) and the monocarboxylate transporter 1 (MCT1). CD13 cleaves N-terminal amino acids from polypeptides and exosome-associated CD13 degrades enkephalins, influencing the activity of ligands of the opioid receptor and thus neuronal cAMP levels. Since these exosomes contain the lactate transporter MCT1 together with glycolytic enzymes, they may serve to deliver energy substrates to neurons (Potolicchio et al., 2005). Moreover, microvesicles carrying the proinflammatory cytokine IL- $1 \beta$ shed from the plasma membrane of microglial cells and astrocytes in response to ATP stimulation and activation of acid sphingomyelinase (Bianco et al., 2009).

In response to oxidative and heat stress, cultured astrocytes release elevated amounts of the heat-shock protein 70 (Hsp/Hsc70) as well as synapsin 1 in association with exosomes (Taylor et al., 2007; Wang et al., 2011). Intriguingly, astrocytederived exosomes have been reported to contain mitochondrial DNA (Guescini et al., 2010). Secretion of microvesicles comprising a mixture of exosomes, shedding vesicles, and possibly also apoptotic bodies is a prominent feature of brain tumor cells, which often originate from astrocytes (van der Vos et al., 2011). Microvesicles derived from highly aggressive glioblastoma multiforme tumors (GBM), carry oncogenic EFGRvIII in addition to immunosuppressive and angiogenic factors (Al-Nedawi et al., 2008; Graner et al., 2009). They are moreover characterized by the presence of nucleic acids, including mRNAs, miRNAs, non-coding RNAs, retrotransposon elements, genomic DNA, and cDNA derived from oncogenic sequences (Skog et al., 2008; Balaj et al., 2011).

\section{FUNCTIONS OF NERVOUS SYSTEM EXOSOMES}

Two general functions have been ascribed to exosome secretion: disposal of unneeded cell components and signaling to neighboring cells involving the horizontal transfer of biomolecules (Lotvall and Valadi, 2007; Simons and Raposo, 2009). Both functional properties assigned to exosomes appear eligible to be utilized by neural cells, which operate as a long-term cellular network relying on finely tuned cell-cell interactions, mutual support, and the clearance of remnants. The physiological and pathological impact of exosomes in the nervous system has been a topic of discussion (Smalheiser, 2007; Aguzzi and Rajendran, 2009), however, we are only beginning to capture a true picture (Figure 2). Exosomes secreted by neurons have been implicated in synaptic plasticity. Enhanced glutamatergic activity within cultures of mature cortical neurons stimulates the release of exosomes carrying the AMPA receptor subunit GluR2 from the somatodendritic postsynaptic compartment (Lachenal et al., 2011). These findings suggest that activity-dependent exosome secretion may help to adapt the efficacy of synaptic transmission by depletion of neurotransmitter receptors from the postsynaptic compartment. What happens to the exosomes after the release, whether they become internalized by the presynaptic neuron or surrounding glial cells remains to be determined. A synaptic transfer of wnt-signaling molecules involving exosome-like vesicles has been shown to occur in drosophila larva at the neuromuscular junction (Koles et al., 2012).

In the context of CNS pathology, it appears that pathogenic proteins such as $\beta$-amyloid peptide, prion protein, $\alpha$-synuclein, tau, and superoxide dismutase are released from cells in association with exosomes (Fevrier et al., 2004; Rajendran et al., 2006; Gomes et al., 2007; Emmanouilidou et al., 2010; Saman et al., 2011). These proteins have in common the activity to form aggregates (amyloids, prionoids) that escape the normal cellular degradation machinery. It is not clear yet whether exosome-associated secretion of amyloidogenic proteins helps to relieve cells of potentially detrimental components, or if the pathogen-filled exosomes engage in propagating neurodegenerative "seeds" within the tissue. Exosomes carrying prions have been shown to be infectious (Fevrier et al., 2004; Vella et al., 2007). However, the processing of pathogenic exosomes by recipient cells remains to be demonstrated.

More is known about the role of membrane vesicles released by glial cells. Microvesicles (including exosomes) are released by glioma cells, which carry the mRNA and protein of oncogenic EGFRvIII as well as angiogenic factors. They can be internalized by surrounding cells, promoting cell transformation or mediating tubular growth of endothelial cells (Al-Nedawi et al., 2008; Skog et al., 2008; Graner et al., 2009; Svensson et al., 2011; van der Vos et al., 2011). Thus, glioma-derived microvesicles appear to modulate the environment to favor tumor growth. Intriguingly, tumor-derived microvesicles can also be detected in the circulation of glioma-bearing mice or human glioblastoma patients demonstrating that they are produced in vivo and are capable of crossing the blood brain barrier. Microvesicle release from primary cortical astrocytes and microglial cells appears to be triggered by ATP-mediated activation of $\mathrm{P}_{2} \mathrm{X}_{7}$ receptors and downstream stimulation of acid sphingomyelinase (Bianco et al., 2009). A recent study suggests that this special type of microvesicle signals to neurons resulting in modulation of synaptic activity (Antonucci et al., 2012). Application of microvesicles isolated from ATP-stimulated microglial cells to neurons in vitro and in vivo gave rise to increased neurotransmission, that appeared to be due to an enhanced release probability at the presynaptic site. However, the contribution of exosome release to these phenomena is unclear. Scanning electron micrograph pictures of glioma cells and fluorescence microscopy analysis of ATP-stimulated glial cells, as well as the heterogeneous size profile of the released microvesicles indicate that shedding from the cell surface is the dominant form of vesicle generation in these paradigms. Nevertheless, it is possible that exosomes account for some of the described functions since they are also present in the vesicular fractions analyzed. Astrocytes release exosomes carrying Hsp70 and synapsin 1 in response to heat or oxidative stress, which have been suggested to ship neuroprotective cargo to neurons facilitating their survival (Taylor et al., 2007). Intriguingly, synapsin 1 is first released from the exosomal cytosolic compartment under these conditions, before it acts on neurons in its soluble form (Wang et al., 2011). 


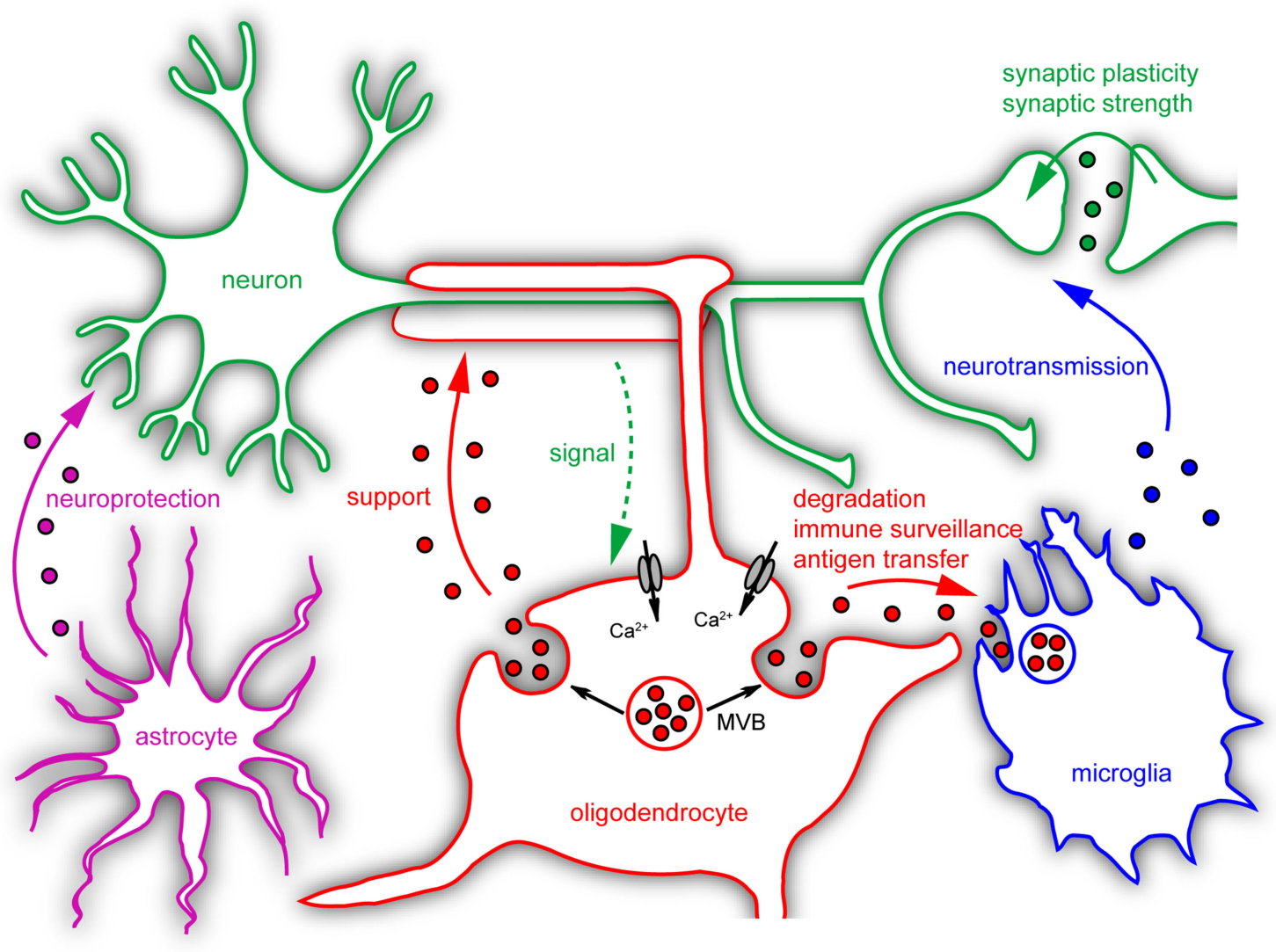

FIGURE 2 | Postulated roles of microvesicles in neural cell communication. Neural cells release different types of microvesicles with several known or suggested functions. Neurons secrete exosomes which may influence synaptic plasticity. Microglia modulate neurotransmission via shedding microvesicles. Astrocyte-derived exosomes carry neuroprotective cargo and could contribute to neuronal survival. Neuronal signals trigger exosome release from oligodendrocytes by raising intracellular $\mathrm{Ca}^{2+}$-levels. Upon internalization by neurons these exosomes could provide support to axons. Microglia take up and degrade oligodendroglial exosomes without changing their inflammatory properties. Under specific pathological conditions these exosomes may transfer antigens to microglial cells or other APCs and induce inflammatory responses.

\section{ROLE OF OLIGODENDROGLIAL EXOSOMES IN CELL COMMUNICATION}

Oligodendrocytes secrete MVB-derived exosomes in a $\mathrm{Ca}^{2+}$ dependent fashion, which also carry mRNA and miRNA in addition to myelin proteins and lipids (Krämer-Albers et al., 2007; Krämer-Albers et al., unpublished observations). These exosomes appear to convey autocrine signals that inhibit membrane expansion and myelin formation by oligodendrocytes in culture in response to neuron-derived trophic factors (Bakhti et al., 2011). This autocrine signaling pathway does not involve exosome reinternalization but is mediated by activation of second messenger cascades involving RhoA, Fyn, and FAK. Furthermore, it has been shown that microglia take up oligodendroglial exosomes by macropinocytosis, which subsequently become degraded and apparently fail to provoke an immune response (Fitzner et al., 2011). Internalization is observed preferentially in MHC class II negative microglia and does not promote microglial activation. The authors suggest that microglia are engaged in the degradation of excess myelin components secreted by the exosome pathway. It will be interesting to determine whether exosomes derived from oligodendrocytes exposed to pathogens or specific forms of stress will be able to induce an inflammatory response. This is particularly relevant to the still unresolved etiology of multiple sclerosis and the question of how CNS self-antigens are transferred to trigger a myelin-specific autoimmune response.

Our recent work indicates that oligodendroglial exosomes play an important role in mutual communication between oligodendrocytes and neurons. We found that neurotransmitter release stimulates oligodendroglial exosome secretion by activating $\mathrm{Ca}^{2+}$ permeable ionotropic receptors on the surface of oligodendrocytes. Furthermore, neurons internalize oligodendroglial exosomes by endocytosis and utilize their cargo (submitted manuscript). These findings suggest that neuronal activity triggers the transfer of oligodendroglial exosomes and their cargo to neurons. Thus, neurons would regulate their supply of glia-derived exosomal proteins, mRNAs, and miRNAs. Myelinated axons exist as long protrusions at a distance from the neuronal cell body and are shielded from the CNS environment by the myelin membrane (Nave, 2010b). Hence, external support by exosome-mediated transfer of glial substrates could be contributing to and be critical for long-term axonal maintenance. A lack of glial support is modeled by PLP and CNP knockout mice, which develop a 
progressive axonal degeneration (Edgar and Nave, 2009). PLP and CNP are both components of oligodendroglial exosomes and thus, it is tempting to speculate about the potential contribution of exosomes to glial support. Oligodendroglial exosomes additionally include substances that can contribute to neuroprotection such as Hsp/Hsc70. There is evidence that the squid giant axon locally picks up Hsps from periaxonal glial cells (Tytell et al., 1986) and it was indeed suggested that the transfer is mediated by exosomes (Tytell, 2005). Moreover, newly synthesized glial RNAs are delivered to the giant axon in response to axonal depolarization (Eyman et al., 2007). In the PNS, it has been described that axons receive ribosomal subunits in association with vesicles from myelinating Schwann cells (Court et al., 2008). It will be interesting to determine, whether oligodendroglial exosomes exhibit neuroprotective or neurotrophic functions.

\section{RELEVANCE FOR MYELIN DISEASE}

Myelin diseases are characterized by a developmental hypo/ dysmyelination or a secondary demyelination. They can be inherited (leukodystrophies) or acquired such as multiple sclerosis. The pathology of multiple sclerosis involves an immune-mediated degeneration of the myelin sheath. Which factors direct the specificity of the immune response toward myelin and whether myelin antigens in multiple sclerosis are processed by APCs is unknown. Exosomes are known to deliver antigens to APCs (Bobrie et al., 2011). In spite of reports that oligodendroglial exosomes appear not to activate microglia, it is feasible that other APCs such as dendritic cells, which invade the CNS under certain conditions, process oligodendroglial exosomes and present the antigens on their surface to initiate an immune response. Future studies will determine whether oligodendroglial exosomes can mediate myelin antigen transfer to dendritic cells. Furthermore, exosome composition may be altered in certain pathological conditions, causing a switch of immunologically inert into immunologically active exosomes. Oligodendroglial exosome composition indeed may be altered in Pelizaeus-Merzbacher disease (PMD) resulting from duplications in the PLP1 gene. Overexpression of PLP leads to its accumulation in late endosomes (Simons et al., 2002) and may yield an increased release of PLP in association with exosomes. Increased exosome release or an altered exosome protein/lipid

\section{REFERENCES}

Aguzzi, A., and Rajendran, L. (2009). The transcellular spread of cytosolic amyloids, prions, and prionoids. Neuron 64, 783-790.

Allen, N. J., and Barres, B. A. (2009). Neuroscience: glia - more than just brain glue. Nature 457, 675-677.

Al-Nedawi, K., Meehan, B., Micallef, J., Lhotak, V., May, L., Guha, A., and Rak, J. (2008). Intercellular transfer of the oncogenic receptor EGFRvIII by microvesicles derived from tumour cells. Nat. Cell Biol. 10, 619-624.

Antonucci, F., Turola, E., Riganti, L., Caleo, M., Gabrielli, M., Perrotta, C.,

stoichiometry may thus trigger inflammatory reactions in the CNS contributing to PMD pathology.

Common to most myelin diseases is the phenomenon of progressive axonal degeneration due to lack of glial support. This secondary neuronal damage is the major cause of irreversible disability and death of the patients. Thus, it is of clinical importance to decipher the potential implications of oligodendroglial exosomes in neuroprotection and to identify the beneficial components, with the ultimate goal to develop therapeutic strategies mitigating axonal degeneration. Furthermore, there is accumulating evidence that exosomes qualify as promising vehicles for the delivery of therapeutic agents into the brain, as they can be delivered across biological barriers such as the blood brain barrier (Lakhal and Wood, 2011).

\section{CONCLUSION}

The hypothesized role of secreted microvesicles/exosomes in neural cell communication is materializing into a picture substantiated by experimental evidence (Figure 2). Microvesicles may execute their functions by distinct modes of action: (1) internalization by target cells and cargo retrieval, (2) binding to the cell surface and triggering second messenger pathways, and (3) release of components into the extracellular matrix. However, their interaction with target cells is not well understood at a mechanistic level. To date, most theories of exosome function have arisen from in vitro data. The field awaits genetic mouse models that interfere with neural exosome secretion to demonstrate the in vivo relevance of exosome-mediated processes. Nonetheless, the versatile role of CNS microvesicles opens up new perspectives for the understanding and treatment of neurodegenerative diseases including diseases of myelin.

\section{ACKNOWLEDGMENTS}

The authors are grateful to Jacqueline Trotter for support and comments on the manuscript. We thank Wiebke Möbius for providing the EM picture and Jesa Amphornrat for figure preparation. We also would like to thank the reviewers for their efforts, which lead to improvement of the manuscript. Our work is supported by the European Leukodystrophy Association, the Intramural Funding Program, and the Focus Program of Translational Neuroscience (FTN) of the Johannes Gutenberg University Mainz.

oncogene sequences. Nat. Commun. 2, 180 .

P. Vlementi, E., Giussani, rio, C. (2012). Microvesicles released from microglia stimulate synaptic activity via enhanced sphingolipid metabolism. EMBO J. 31, 1231-1240.

Bakhti, M., Winter, C., and Simons, M. (2011). Inhibition of myelin membrane sheath formation by oligodendrocyte-derived exosomelike vesicles. J. Biol. Chem. 286, 787-796.

Balaj, L., Lessard, R., Dai, L., Cho, Y. J., Pomeroy, S. L., Breakefield, X. O., and Skog, J. (2011). Tumour microvesicles contain retrotransposon elements and amplified
Bianco, F., Perrotta, C., Novellino, L., Francolini, M., Riganti, L., Menna, E., Saglietti, L., Schuchman, E. H., Furlan, R., Clementi, E., Matteoli, M., and Verderio, C. (2009). Acid sphingomyelinase activity triggers microparticle release from glial cells. EMBO J. 28, 1043-1054.

Bobrie, A., Colombo, M., Raposo, G., and Thery, C. (2011). Exosome secretion: molecular mechanisms and roles in immune responses. Traffic 12, 1659-1668.

Buschow, S. I., Nolte-'t Hoen, E. N., van Niel, G., Pols, M. S., ten Broeke, T.,
Lauwen, M., Ossendorp, F., Melief, C. J., Raposo, G., Wubbolts, R., Wauben, M. H., and Stoorvogel, W. (2009). MHC II in dendritic cells is targeted to lysosomes or $\mathrm{T}$ cellinduced exosomes via distinct multivesicular body pathways. Traffic 10 , 1528-1542.

Camussi, G., Deregibus, M. C., Bruno, S., Cantaluppi, V., and Biancone, L. (2010). Exosoes/microvesicles as a mechanism of cell-to-cell communication. Kidney Int. 78, 838-848.

Cocucci, E., Racchetti, G., and Meldolesi, J. (2009). Shedding microvesicles: artefacts no more. Trends Cell Biol. 19, 43-51. 
Court, F. A., Hendriks, W. T., MacGillavry, H. D., Alvarez, J., and van Minnen, J. (2008). Schwann cell to axon transfer of ribosomes: toward a novel understanding of the role of glia in the nervous system. $J$. Neurosci. 28, 11024-11029.

Edgar, J. M., and Nave, K. A. (2009). The role of CNS glia in preserving axon function. Curr. Opin. Neurobiol. 19, 498-504.

Emmanouilidou, E., Melachroinou, K., Roumeliotis, T., Garbis, S. D., Ntzouni, M., Margaritis, L. H., Stefanis, L., and Vekrellis, K. (2010). Cell-produced alpha-synuclein is secreted in a calcium-dependent manner by exosomes and impacts neuronal survival. J. Neurosci. 30, 6838-6851.

Eyman, M., Cefaliello, C., Ferrara, E., De Stefano, R., Lavina, Z. S., Crispino, M., Squillace, A., van Minnen, J., Kaplan, B. B., and Giuditta, A. (2007). Local synthesis of axonal and presynaptic RNA in squid model systems. Eur. J. Neurosci. 25, 341-350.

Faure, J., Lachenal, G., Court, M., Hirrlinger, J., Chatellard-Causse, C., Blot, B., Grange, J., Schoehn, G., Goldberg, Y., Boyer, V., Kirchhoff, F., Raposo, G., Garin, J., and Sadoul, R. (2006). Exosomes are released by cultured cortical neurones. Mol. Cell. Neurosci. 31, 642-648.

Fevrier, B., Vilette, D., Archer, F., Loew, D., Faigle, W., Vidal, M., Laude, H., and Raposo, G. (2004). Cells release prions in association with exosomes. Proc. Natl. Acad. Sci. U.S.A. 101, 9683-9688.

Fitzner, D., Schnaars, M., van Rossum, D., Krishnamoorthy, G., Dibaj, P., Bakhti, M., Regen, T., Hanisch, U. K., and Simons, M. (2011). Selective transfer of exosomes from oligodendrocytes to microglia by macropinocytosis. J. Cell. Sci. 124, 447-458.

Gomes, C., Keller, S., Altevogt, P., and Costa, J. (2007). Evidence for secretion of $\mathrm{Cu}, \mathrm{Zn}$ superoxide dismutase via exosomes from a cell model of amyotrophic lateral sclerosis. $\mathrm{Neu}$ rosci. Lett. 428, 43-46.

Graner, M. W., Alzate, O., Dechkovskaia, A. M., Keene, J. D., Sampson, J. H., Mitchell, D. A., and Bigner, D. D. (2009). Proteomic and immunologic analyses of brain tumor exosomes. FASEB J. 23, 1541-1557.

Guescini, M., Genedani, S., Stocchi, V., and Agnati, L. F. (2010). Astrocytes and glioblastoma cells release exosomes carrying mtDNA. J. Neural Transm. 117, 1-4.

Hsu, C., Morohashi, Y., Yoshimura, S., Manrique-Hoyos, N., Jung, S.,
Lauterbach, M. A., Bakhti, M., Gronborg, M., Mobius, W., Rhee, J., Barr F. A., and Simons, M. (2010). Regulation of exosome secretion by Rab35 and its GTPase-activating proteins TBC1D10A-C. J. Cell Biol. 189, 223-232.

Kettenmann, H., Hanisch, U. K., Noda, M., and Verkhratsky, A. (2011). Physiology of microglia. Physiol. Rev. 91, 461-553.

Koles, K., Nunnari, J., Korkut, C., Barria, R., Brewer, C., Li, Y., Leszyk, J., Zhang, B., and Budnik, V. (2012). Mechanism of Evi-exosome release at synaptic boutons. J. Biol. Chem. doi: $\quad 10.1074 /$ jbc.M112.342667. [Epub ahead of print].

Krämer, E. M., Koch, T., Niehaus, A., and Trotter, J. (1997). Oligodendrocytes direct glycosyl phosphatidylinositolanchored proteins to the myelin sheath in glycosphingolipid-rich complexes. J. Biol. Chem. 272, 8937-8945.

Krämer-Albers, E. M., Bretz, N., Tenzer, S., Winterstein, C., Mobius, W., Berger, H., Nave, K. A., Schild, H., and Trotter, J. (2007). Oligodendrocytes secrete exosomes containing major myelin and stress-protective proteins: trophic support for axons? Proteomics Clin. Appl. 1, 1446-1461. Lachenal, G., Pernet-Gallay, K., Chivet, M., Hemming, F. J., Belly, A., Bodon, G., Blot, B., Haase, G., Goldberg, Y., and Sadoul, R. (2011). Release of exosomes from differentiated neurons and its regulation by synaptic glutamatergic activity. Mol. Cell. Neurosci. 46, 409-418.

Lakhal, S., and Wood, M. J. (2011). Exosome nanotechnology: an emerging paradigm shift in drug delivery: exploitation of exosome nanovesicles for systemic in vivo delivery of RNAi heralds new horizons for drug delivery across biological barriers. Bioessays 33, 737-741.

Lakkaraju, A., and Rodriguez-Boulan, E. (2008). Itinerant exosomes: emerging roles in cell and tissue polarity. Trends Cell Biol. 18, 199-209.

Lotvall, J., and Valadi, H. (2007). Cell to cell signalling via exosomes through esRNA. Cell Adh. Migr. 1, 156-158.

Mathivanan, S., Fahner, C. J., Reid, G. E., and Simpson, R. J. (2012). ExoCarta 2012: database of exosomal proteins, RNA and lipids. Nucleic Acids Res. 40, D1241-D1244.

Nave, K. A. (2010a). Myelination and support of axonal integrity by glia. Nature 468, 244-252.

Nave, K. A. (2010b). Myelination and the trophic support of long axons. Nat. Rev. Neurosci. 11, 275-283.
Ostrowski, M., Carmo, N. B., Krumeich, S., Fanget, I., Raposo, G., Savina, A., Moita, C. F., Schauer, K. Hume, A. N., Freitas, R. P., Goud, B., Benaroch, P., Hacohen, N., Fukuda, M., Desnos, C., Seabra, M. C., Darchen, F., Amigorena, S., Moita, L. F., and Thery, C. (2010). Rab27a and Rab27b control different steps of the exosome secretion pathway. Nat. Cell Biol. 12, 19-30; sup 1-13.

Pegtel, D. M., Cosmopoulos, K., Thorley-Lawson, D. A., van Eijndhoven, M. A., Hopmans, E. S. Lindenberg, J. L., de Gruijl, T. D., Wurdinger, T., and Middeldorp, J. M. (2010). Functional delivery of viral miRNAs via exosomes. Proc. Natl. Acad. Sci. U.S.A. 107, 6328-6333.

Potolicchio, I., Carven, G. J., Xu, X., Stipp, C., Riese, R. J., Stern, L. J., and Santambrogio, L. (2005). Proteomic analysis of microglia-derived exosomes: metabolic role of the aminopeptidase CD13 in neuropeptide catabolism. J. Immunol. 175, 2237-2243.

Rajendran, L., Honsho, M., Zahn, T. R. Keller, P., Geiger, K. D., Verkade, P. and Simons, K. (2006). Alzheimer's disease beta-amyloid peptides are released in association with exosomes. Proc. Natl. Acad. Sci. U.S.A. 103, 11172-11177.

Saman, S., Kim, W., Raya, M., Visnick, Y., Miro, S., Saman, S., Jackson, B., McKee, A. C., Alvarez, V. E., and Lee, N. C. Hall, G. F. (2011). Exosomeassociated tau is secreted in tauopathy models and is selectively phosphorylated in cerebrospinal fluid (CSF) in early Alzheimer's disease. J. Biol. Chem. 287, 3842-3849.

Simons, M., Krämer, E. M., Macchi, P., Rathke-Hartlieb, S., Trotter, J., Nave, K. A., and Schulz, J. B. (2002). Overexpression of the myelin proteolipid protein leads to accumulation of cholesterol and proteolipid protein in endosomes/lysosomes: implications for Pelizaeus-Merzbacher disease. J. Cell Biol. 157, 327-336.

Simons, M., and Raposo, G. (2009). Exosomes - vesicular carriers for intercellular communication. Curr. Opin. Cell Biol. 21, 575-581.

Simpson, R. J., Jensen, S. S., and Lim, J. W. (2008). Proteomic profiling of exosomes: current perspectives. Proteomics 8, 4083-4099.

Skog, J., Wurdinger, T., van Rijn, S., Meijer, D. H., Gainche, L., SenaEsteves, M., Curry, W. T. Jr., Carter, B. S., Krichevsky, A. M., and Breakefield, X. O. (2008). Glioblastoma microvesicles transport RNA and proteins that promote tumour growth and provide diagnostic biomarkers. Nat. Cell Biol. 10, 1470-1476.

Smalheiser, N. R. (2007). Exosomal transfer of proteins and RNAs at synapses in the nervous system. Biol. Direct 2, 35.

Svensson, K. J., Kucharzewska, P., Christianson, H. C., Skold, S., Lofstedt, T., Johansson, M. C., Morgelin, M., Bengzon, J., Ruf, W., and Belting, M. (2011). Hypoxia triggers a proangiogenic pathway involving cancer cell microvesicles and PAR-2-mediated heparin-binding EGF signaling in endothelial cells. Proc. Natl. Acad. Sci. U.S.A. 108, 13147-13152.

Taylor, A. R., Robinson, M. B., Gifondorwa, D. J., Tytell, M., and Milligan, C. E. (2007). Regulation of heat shock protein 70 release in astrocytes: role of signaling kinases. Dev. Neurobiol. 67, 1815-1829.

Thery, C. (2011). Exosomes: secreted vesicles and intercellular communications. F1000 Biol. Rep. 3, 15

Thery, C., Ostrowski, M., and Segura, E. (2009). Membrane vesicles as conveyors of immune responses. Nat. Rev. Immunol. 9, 581-593.

Trajkovic, K., Hsu, C., Chiantia, S., Rajendran, L., Wenzel, D., Wieland, F., Schwille, P., Brugger, B., and Simons, M. (2008). Ceramide triggers budding of exosome vesicles into multivesicular endosomes. Science 319, 1244-1247.

Tytell, M. (2005). Release of heat shock proteins (Hsps) and the effects of extracellular Hsps on neural cells and tissues. Int. J. Hyperthermia 21, 445-455.

Tytell, M., Greenberg, S. G., and Lasek, R. J. (1986). Heat shock-like protein is transferred from glia to axon. Brain Res. 363, 161-164.

Valadi, H., Ekstrom, K., Bossios, A., Sjostrand, M., Lee, J. J., and Lotvall, J. O. (2007). Exosome-mediated transfer of mRNAs and microRNAs is a novel mechanism of genetic exchange between cells. Nat. Cell Biol. 9, 654-659.

van der Vos, K. E., Balaj, L., Skog, J., and Breakefield, X. O. (2011). Brain tumor microvesicles: insights into intercellular communication in the nervous system. Cell. Mol. Neurobiol. 31, 949-959.

van Niel, G., Porto-Carreiro, I., Simoes, S., and Raposo, G. (2006). Exosomes: a common pathway for a specialized function. J. Biochem. 140, 13-21.

Vella, L. J., Greenwood, D. L., Cappai, R., Scheerlinck, J. P., and Hill, A. F. (2008). Enrichment of prion protein in exosomes derived from ovine 
cerebral spinal fluid. Vet. Immunol. Immunopathol. 124, 385-393.

Vella, L. J., Sharples, R. A., Lawson, V. A., Masters, C. L., Cappai, R., and Hill, A. F. (2007). Packaging of prions into exosomes is associated with a novel pathway of PrP processing. J. Pathol. 211, 582-590.

Wang, S., Cesca, F., Loers, G., Schweizer, M., Buck, F., Benfenati, F., Schachner, M., and Kleene, R. (2011). Synapsin $\mathrm{I}$ is an oligomannose-carrying glycoprotein, acts as an oligomannose-binding lectin, and promotes neurite outgrowth and neuronal survival when released via glia-derived exosomes. J. Neurosci. 31, 7275-7290.

Zhang, Y., Liu, D., Chen, X., Li, J., Li, L., Bian, Z., Sun, F., Lu, J., Yin, Y., Cai, X., Sun, Q., Wang, K., Ba, Y., Wang, Q., Wang, D., Yang, J., Liu, P., Xu, T., Yan, Q., Zhang, J., Zen, K., and Zhang, C. Y. (2010). Secreted monocytic miR150 enhances targeted endothelial cell migration. Mol. Cell 39, 133-144.
Conflict of Interest Statement: The authors declare that the research was conducted in the absence of any commercial or financial relationships that could be construed as a potential conflict of interest.

Received: 15 February 2012; paper pending published: 21 March 2012; accepted: 11 April 2012; published online: 30 April 2012.

Citation: Frühbeis $C$, Fröhlich $D$ and Krämer-Albers E-M (2012) Emerging roles of exosomes in neuron-glia communication. Front. Physio. 3:119. doi: 10.3389/fphys.2012.00119

This article was submitted to Frontiers in Membrane Physiology and Biophysics, a specialty of Frontiers in Physiology.

Copyright $@ 2012$ Frühbeis, Fröhlich and Krämer-Albers. This is an open-access article distributed under the terms of the Creative Commons Attribution Non Commercial License, which permits noncommercial use, distribution, and reproduction in other forums, provided the original authors and source are credited. 